Gazi University
Journal of Science
$\mathrm{http} / /$ dergipark.gov.tr/gujs

\title{
Common Solution for Nonlinear Operators in Banach Spaces
}

\author{
Enyinnaya EKUMA-OKEREKE ${ }^{1, *(1)}$, Felix Moibi OKORO² \\ ${ }^{I}$ Department of Mathematics, Federal University of Petroleum Resources, Effurun, Nigeria \\ ${ }^{2}$ Department of Mathematics, Ambrose Alli University, Ekpoma, Nigeria
}

\section{Highlights}

- Construction of a hybrid inertial algorithm.

- Theoretical proof of the constructed algorithm.

- Numerical illustration of the applicability of the algorithm.

\section{Article Info}

Received: 10/07/2019

Accepted: 03/04/2020

Keywords

Bregman nonexpansive-type operator

Common element

Continuous monotone operator

Inertial component

\begin{abstract}
This paper formulates a hybrid approximation process involving inertial component and demonstrates a convergence results for it. The formulated scheme converges faster and finds a common solution for some nonlinear operators in Banach spaces. The method of our proof and results obtained is well involved and significant.
\end{abstract}

\section{INTRODUCTION}

Throughout, the Banach space $X$ is taken to be real and reflexive with $X^{*}$ as the dual. We let $\|\|:. X \rightarrow R$ represent the norm function. Let $d_{h}: d o m h \times \operatorname{int}(d o m h) \rightarrow R^{+}$represent a bifunctions induced by a convex function. Let $d o m h=\{u \in X: h(u)<+\infty\}$ and $\operatorname{int}(d o m h)$ represent the domain and interior domain of a convex function, $h: X \rightarrow(-\infty,+\infty]$ respectively. The convex function $h$ is Gâteaux differentiable at $u$ if $\lim _{s \rightarrow 0^{+}} \frac{(h(u+s z)-h(u))}{s}=h^{\circ}(u, z)$ exists for any $z$ in $X$. By this, $h^{\circ}(u, z)=\nabla h(u)$, as the gradient of $h$.

Let the convex function $h$ be Gâteaux differentiable at $u$, then $d_{h}: \operatorname{dom} h \times \operatorname{int}(\operatorname{dom} h) \rightarrow R^{+}$defined by

$$
d_{h}(z, u)=h(z)-h(u)-\langle\nabla h(u), z\rangle+\langle\nabla h(u), u\rangle,
$$

is the Bregman function induce by $h$.

This function $d_{h}: d o m h \times \operatorname{int}(d o m h) \rightarrow R^{+}$defined by (1) has some nice properties like:

$\mathrm{P} 1$. The function $d_{h}(., u)$ is convex. 
P2. $d_{h}(u, u)=0$.

P3. $d_{h}(z, u)>0$.

P4. $d_{h}(z, u)=d_{h}(z, v)+d_{h}(v, u)+\langle z-v, \nabla h(v)\rangle-\langle z-v, \nabla h(u)\rangle$.

P5. $d_{h}(u, v)+d_{h}(v, u)=\langle u-v, \nabla h(u)\rangle-\langle u-v, \nabla h(v)\rangle$.

P6. $d_{h}(u, v) \leq\|u\|\|\nabla h(u)-\nabla h(v)\|+\|v\|\|\nabla h(u)-\nabla h(v)\|$.

Observe that P4 implies P5 and P6 if $u=z$. For proof of (P1 - P3), see [1,2].

Let $K$ represent a non-void, closed, convex subset of $\operatorname{int}($ domh $)$. Let $G: K \rightarrow K$ represent a map. $G: K \rightarrow K$ is nonexpansive if $\|G u-G z\| \leq\|u-z\|, \forall u, z \in K ; G: K \rightarrow K$ is (quasi)-nonexpansive if $\left\|G u-z^{0}\right\| \leq\left\|u-z^{0}\right\|$, and $F i x(G)=\left\{z^{0} \in K: G u=u\right\}$ is the collection of fixed point of $G: K \rightarrow K$. An element $u^{*} \in K$ is asymptotic fixed point of $G: K \rightarrow K$ when $\left\{u_{n}\right\}$ is contained in $K$ and converging weakly to $u$ so that $\left\|u_{n}-G u_{n}\right\|=0$. We denote the set by $F i \hat{x}(G)$.

A map $G: K \rightarrow$ int $d o m h$, is Bregman relatively nonexpansive (BRNE) [3] if

$$
\begin{aligned}
& d_{h}\left(z^{0}, G u\right) \leq d_{h}\left(z^{0}, u\right), \forall u \in K, \forall z^{0} \in F i x(G) \\
& F i \hat{x}(G)=F i x(G) .
\end{aligned}
$$

For a differentiable function $h: X \rightarrow R^{+}$and for all $u \in X$, [4,5] gives

$$
d_{h}\left(u_{0}, u\right)=\min \left\{d_{h}(z, u): u \in X\right\} \Leftrightarrow\left\langle\nabla h(u), z-u_{0}\right\rangle-\left\langle\nabla h\left(u_{0}\right), z-u_{0}\right\rangle \leq 0, \forall z \in K .
$$

In addition, if $K \subset X$, then for $u \in \operatorname{int} d o m h$, we have a unique $u_{0} \in K$ such that the mapping $P_{K}^{h}:$ int $d o m h \rightarrow K$ which satisfy

$$
d_{h}\left(u_{0}, u\right)=\min \left\{d_{h}(z, u): z \in K\right\}
$$

is the Projection of $u \in$ int $d o m h$ onto the set $K \subset d o m h$, where $P_{K}^{h}(u)=u_{0}$. The Bregman Projection mapping in view of $[4,5]$ satisfy:

$$
d_{h}\left(z, P_{K}^{h}(u)\right)+d_{h}\left(P_{K}^{h}(u), u\right) \leq d_{h}(z, u), \quad z \in K .
$$

Given $h$ a norm square with $u \in X$, then we see that $\nabla h(u)=2 J u$, where $J: X \rightarrow X^{*}$ is defined and (1) reduce to $\phi(z, u)=\|z\|^{2}-2\langle J u, z\rangle+\|u\|^{2}$ known as the Lyapunov functional [6].

The function $h: X \rightarrow(-\infty,+\infty]$ is Legendre [7], if the following hold

(i) int dom $h$ is non-void, $h$ is differentiable on int $d o m h$ with $d o m h=$ int $d o m h$,

(ii) int $d o m h^{*}$ is non-void, $h^{*}$ is differentiable on int $d o m h^{*}$ with $d o m h^{*}=$ int $d o m h^{*}$.

With $h: X \rightarrow(-\infty,+\infty]$ a Legendre function, and $X$ reflexive, then $\nabla h$ is a bijection which satisfies $\nabla h=\left(\nabla h^{*}\right)^{-1}$, range $\nabla h=$ domain $\nabla h^{*}=$ int domain $h^{*}$. If $h: X \rightarrow(-\infty,+\infty]$ is single-valued and $X$ 
is smooth and strictly convex, then $J=\nabla h$. Given $h(u)=t^{-1}\|u\|^{2}, t \in(1, \infty)$, then we have a Legendre function [8-12].

The modulus of total convexity of $h$ at $u \in \operatorname{int} \operatorname{domh} W_{h}(u,):$. int $d o m h \times R^{+} \rightarrow R^{+}$is defined by

$$
W_{h}(u, s)=\inf \left\{d_{h}(z, u): z \in d o m h,\|z-u\|=s\right\}
$$

If $W_{h}(x, s)$ is positive, then $h: X \rightarrow(-\infty,+\infty]$ becomes totally convex at $u$ for positive values of $s$. For more information, see [7].

A mapping $a: K \rightarrow X^{*}$ is monotone if $\forall u, z \in K$, we have

$$
\langle a u, u-z\rangle-\langle a z, u-z\rangle \geq 0
$$

Note that a point $z^{0} \in K$ such that

$$
\left\langle a z^{0}, z\right\rangle-\left\langle a z^{0}, z^{0}\right\rangle \geq 0 \quad \forall z \in K,
$$

solves a variational inequality problem. The collection of solution of (7) is represented by

$$
V I(K, a)=\left\{z^{0} \in K:\left\langle a z^{0}, z\right\rangle-\left\langle a z^{0}, z^{0}\right\rangle \geq 0 \quad \forall z \in K\right\}
$$

$$
\begin{aligned}
& \text { Suppose in } \quad \text { addition } \quad a: K \rightarrow X^{*} \text { is continuous, } \quad \text { we } \quad \text { have } \\
& V I(K, a)=\left\{z^{0} \in K:\left\langle a z^{0}, z\right\rangle-\left\langle a z^{0}, z^{0}\right\rangle \geq 0 \quad \forall z \in K\right\} \text { is closed and convex. }
\end{aligned}
$$

Over the years, smooth convex minimization problem involving generalized nonexpansive and Bregman nonexpansive operators has attracted the interest of many researchers and authors seeking for existence of solutions. It is a fact that most published works on these operators has been the iterative approximation of (common) solution of operators. Furthermore, most of these results only focused on the strong convergence of the formulated schemes to the fixed point sets (see [13-15]). However, very few authors have paid attention to the speed or the rate of convergence of sequence of iterates of Bregman nonexpansive-type operators to their (common) fixed point sets when they exists. Thus, a two-step iterative method to increase the rate of convergence was used in the works of [16-18] and which is defined as

$$
u_{n+1}=u_{n}+\beta_{n}\left(u_{n}-u_{n-1}\right)
$$

for all non-negative integers $n$, where $\beta_{n} \in(0,1)$. We note here that the inertial is represented by the component, $\beta_{n}\left(u_{n}-u_{n-1}\right)$.

Using Lyapunov functional, [14] formulated the hybrid method as given below: 


$$
\left\{\begin{array}{l}
u_{0} \in K_{1}=K \\
\hat{y}^{n}=J^{*}\left(b_{n} J\left(u_{n}\right)+\left(1-b_{n}\right) J G J_{r_{n}} u_{n}\right), \\
C_{n}=\left\{u^{*} \in K: \phi\left(u^{*}, \hat{y}^{n}\right) \leq \phi\left(u^{*}, u_{n}\right)\right\} \\
Q_{n}=\left\{u^{*} \in K:\left\langle u_{n}-u^{*}, J u_{0}\right\rangle-\left\langle u_{n}-u^{*}, J u_{n}\right\rangle \geq 0\right\}, \\
u_{n+1}=\Pi_{C_{n} \cap Q_{n}}\left(u_{0}\right),
\end{array}\right.
$$

where $G: K \rightarrow K$ is relatively nonexpansive map. $J_{r}=(J+r M)^{-1} J$ is a resolvent for maximal map $M: K \rightarrow X^{*}$ with $r$ positive. They showed that their method converge strongly to mutual element of $\operatorname{Fix}(G) \cap M^{-1}(0)$ nearest $u_{0}$.

In 2018, [16], formulated and studied the following methods generated by $\left\{u_{n}\right\}$ as follows: $u_{0}, u_{1} \in X$ and

$$
\left\{\begin{array}{l}
K_{0}=X \\
z_{n}=u_{n}-\alpha_{n}\left(u_{n-1}-u_{n}\right), \\
\hat{y}^{n}=J^{*}\left((1-\beta) J\left(z_{n}\right)+\beta J G z_{n}\right), \\
C_{n+1}=\left\{u^{*} \in K: \phi\left(u^{*}, \hat{y}^{n}\right) \leq \phi\left(u^{*}, z_{n}\right)\right\}, \\
u_{n+1}=\Pi_{C_{n+1}}\left(u_{0}\right),
\end{array}\right.
$$

where $G: X \rightarrow X$ is relatively nonexpansive map expressed as

$G u=J^{-1}\left(\sum_{i=1}^{\infty} \eta_{i}\left(\beta_{i} J G x+\left(1-\beta_{i}\right) J G_{i} u\right)\right)$. They showed that their method converge strongly to a mutual element of $\operatorname{Fix}(G)=\bigcap_{i=1}^{\infty} \operatorname{Fix}\left(G_{i}\right)$.

Both algorithms (9) and (10) were formulated in uniformly convex and smooth Banach spaces. We observe also that algorithm (9) combined the intersection of two half sets and at each iteration, it is used and taken as the next Projection which is not easily done in application. Secondly, it has no inertial component that could speed up the convergence of their algorithm. On the other hand, algorithm (10) has the inertial component.

Question. Can we formulate iterative scheme following hybrid method with inertial component without the intersection of two half sets? Can our algorithm converge to common element of our non-void set faster in reflexive and real Banach space?

Our motivation for study is the results of [14] and [16]. We aim to study an iterative scheme with inertial component for our operator. The formulated scheme converges faster and finds a common solution for some nonlinear operators in Banach spaces. The method of our proof and results obtained is well involved and significant.

\section{MAIN RESULTS}

Let $K$ be a non-void, closed and convex subset of reflexive Banach space $X$. Let the function $h: X \rightarrow(-\infty,+\infty)$ represent bounded Legendre, uniform Fréchet differentiable, totally convex. Let the map $G: K \rightarrow K$ represent a Bregman relatively nonexpansive, $a: K \rightarrow X^{*}$ represent a continuous monotone map. We assume $F=\operatorname{Fix}(G) \cap V I(K, a)$ to be non-void. For $u$ element of $X$, define the mapping $T_{r}^{a}: X \rightarrow K$ as follows: 
$T_{r}^{a} x=\left\{z^{0} \in K:\left\langle a z^{0}, z\right\rangle r_{n}-\left\langle a z^{0}, z^{0}\right\rangle r_{n}+\left\langle\nabla h\left(z^{0}\right)-\nabla h(u), z-z^{0}\right\rangle \geq 0, \forall z \in K\right.$

Set $x_{0}, x_{1} \in K$. Then define $\left\{x_{n}\right\}$ by the manner below:

$$
\left\{\begin{array}{l}
x_{0} \in K_{0}=K \\
z_{n}=\nabla h^{*}\left(\nabla h\left(x_{n}\right)+\alpha_{n}\left(\nabla h\left(x_{n}\right)-\nabla h\left(x_{n-1}\right)\right)\right) \\
y_{n}=\nabla h^{*}\left((1-\eta) \nabla h\left(z_{n}\right)+\eta \nabla h\left(G z_{n}\right)\right) \\
w_{n}=T_{r_{n}}^{a} y_{n} \\
K_{n+1}=\left\{u \in K_{n}: d_{h}\left(u, w_{n}\right) \leq d_{h}\left(u, z_{n}\right)\right\} \\
x_{n+1}=P_{K_{n+1}}^{h}\left(x_{0}\right)
\end{array}\right.
$$

where $\left\{r_{n}\right\} \subset(0, \infty), n \in N, \alpha_{n} \in(0,1), \eta \in(0,1)$.

Lemma 2.1. The scheme (11) is well defined.

\section{Proof.}

First, we demonstrate that $F=\operatorname{Fix}(G) \bigcap V I(K, a)$ is closed, convex. Note in [2], $\operatorname{Fix}(G)$ is closed, convex. Also note in [13], $\operatorname{VI}(a, K)$ is closed, convex. So $F=\operatorname{Fix}(T) \cap V I(K, a)$ is closed, convex. Secondly, we demonstrate that $K_{n}$ is closed, convex for each non-negative integer.

To realize this, from our setting in (11), $K_{n}$ is closed. Moreover, since $d_{h}\left(u, w_{n}\right) \leq d_{h}\left(u, z_{n}\right)$ is equivalent of $\left\langle\nabla h\left(z_{n}\right)-\nabla h\left(w_{n}\right), u\right\rangle+\left\langle\nabla h\left(z_{n}\right)-\nabla h\left(w_{n}\right), w_{n}-z_{n}\right\rangle \leq h\left(w_{n}\right)-h\left(z_{n}\right)$,

it follows that $K_{n}$ is a half space and hence convex for each nonnegative integer.

In addition, we demonstrate that $F \subset K_{n}$ for each nonnegative integer. Clearly, from our setting, $F \subset K_{0}=K$. If $F \subset K_{t}$ for some $t>0$, then with $q \in F$, and using P1 together with [13], we obtain

$$
\begin{gathered}
d_{h}\left(q, w_{t}\right)=d_{h}\left(q, T_{r_{n}}^{a} y_{t}\right) \\
\leq d_{h}\left(q, y_{t}\right) .
\end{gathered}
$$

Furthermore,

$$
\begin{aligned}
d_{h}\left(q, y_{t}\right) & =d_{h}\left(q, \nabla h^{*}\left((1-\eta) \nabla h\left(z_{t}\right)+\eta \nabla h\left(G z_{t}\right)\right)\right) \\
& \leq(1-\eta) d_{h}\left(q, z_{t}\right)+\eta d_{h}\left(q, G z_{t}\right) \\
& \leq(1-\eta) d_{h}\left(q, z_{t}\right)+\eta d_{h}\left(q, z_{t}\right) \\
& =d_{h}\left(q, z_{t}\right) .
\end{aligned}
$$

Thus,

$$
d_{h}\left(q, y_{t}\right) \leq d_{h}\left(q, z_{t}\right)
$$

Using (13) in (12) gives 
$d_{h}\left(p, w_{h}\right) \leq d_{h}\left(p, z_{h}\right)$

So $q \in K_{t+1}$ and $K_{t+1} \subset K_{t}$. This implies $F \subset K_{n}$. Thus, (11) become well defined

Lemma 2.2. Let $K$ be a non-void, closed and convex subset of reflexive Banach space $X$. Let the function $h: X \rightarrow(-\infty,+\infty)$ represent bounded Legendre, uniform Fréchet differentiable, totally convex. Let the map $G: K \rightarrow K$ represent a Bregman relatively nonexpansive, $a: K \rightarrow X^{*}$ represent a continuous monotone map. We assume $F=\operatorname{Fix}(G) \cap \operatorname{VI}(K, a)$ to be non-void. Let $\left\{x_{n}\right\}$ be produced by (11). Then the following holds

(i) $\quad \lim _{n \rightarrow \infty}\left\|x_{n+1}-x_{n}\right\|=0$,

(ii) $\quad \lim _{n \rightarrow \infty}\left\|x_{n}-z_{n}\right\|=0$,

(iii) $\quad \lim _{n \rightarrow \infty}\left\|x_{n+1}-w_{n}\right\|=0$,

(iv) $\quad \lim _{n \rightarrow \infty}\left\|z_{n}-w_{n}\right\|=0$,

(v) $\quad \lim _{n \rightarrow \infty}\left\|w_{n}-y_{n}\right\|=0$,

(vi) $\quad \lim _{n \rightarrow \infty}\left\|x_{n}-y_{n}\right\|=0$,

(vii) $\quad \lim _{n \rightarrow \infty}\left\|z_{n}-T z_{n}\right\|=0$.

\section{Proof.}

Seeing that $x_{n}=P_{K_{n}}^{h}\left(x_{0}\right)$ with $x_{n+1}=P_{K_{n+1}}^{h}\left(x_{0}\right) \in K_{n+1} \subset K_{n}$, gives

$$
\begin{aligned}
& d_{h}\left(x_{n}, x_{0}\right) \leq d_{h}\left(x_{n+1}, x_{0}\right)-d_{h}\left(x_{n+1}, x_{n}\right) \\
& d_{h}\left(x_{n+1}, x_{0}\right) \geq d_{h}\left(x_{n}, x_{0}\right) .
\end{aligned}
$$

This shows that $\left\{d_{h}\left(x_{n}, x_{0}\right)\right\}$ is monotone nondecreasing sequence. Besides, from (4),

$$
\begin{aligned}
& d_{h}\left(x_{n}, x_{0}\right)=d_{h}\left(P_{K_{n}}^{h}\left(x_{0}\right), x_{0}\right) \leq d_{h}\left(q, x_{0}\right)-d_{h}\left(q, P_{K_{n}}^{h}\left(x_{0}\right)\right) \leq d_{h}\left(q, x_{0}\right) \quad \forall n \in N \cup\{0\}, q \in F . \\
& \Rightarrow d_{h}\left(x_{n}, x_{0}\right) \leq d_{h}\left(q, x_{0}\right) .
\end{aligned}
$$

This demonstrates boundedness of $\left\{d_{h}\left(x_{n}, x_{0}\right)\right\}$. From [4], boundedness of $\left\{x_{n}\right\}$ hold. But (14) combined with (15), shows that $\lim _{n \rightarrow \infty} d_{h}\left(x_{n}, x_{0}\right)$ exist. Now wlog, let

$\lim _{n \rightarrow \infty} d_{h}\left(x_{n}, x_{0}\right)=l$

In addition to (16) and (4), we get

$$
\begin{aligned}
d_{h}\left(x_{n+\mu}, x_{n}\right)=d_{h}\left(x_{n+\mu}, P_{K_{n}}^{h}\left(x_{0}\right)\right) & \\
& \leq d_{h}\left(x_{n+\mu}, x_{0}\right)-d_{h}\left(x_{n}, x_{0}\right) \rightarrow 0 \text { as } n \rightarrow \infty .
\end{aligned}
$$

So that 


$$
\lim _{n \rightarrow \infty} d_{h}\left(x_{n+\mu}, x_{n}\right)=0, \mu>0 .
$$

In particular,

$\lim _{n \rightarrow \infty} d_{h}\left(x_{n+1}, x_{n}\right)=0$

Therefore, we get from [7]

$\lim _{n \rightarrow \infty}\left\|x_{n+1}-x_{n}\right\|=0$.

This establishes (i).

Now, since $\nabla h$ is uniformly continuous, we get

$\lim _{n \rightarrow \infty}\left\|\nabla h\left(x_{n+1}\right)-\nabla h\left(x_{n}\right)\right\|=0$

Furthermore, from the definition of $z_{n}$, and together with (19), we obtain

$$
\begin{aligned}
\left\|\nabla h\left(x_{n}\right)-\nabla h\left(z_{n}\right)\right\| & =\left\|\nabla h\left(x_{n}\right)-\nabla h\left(x_{n}\right)-\alpha_{n} \nabla h\left(x_{n}-x_{n-1}\right)\right\| \\
& =\left\|\alpha_{n} \nabla h\left(x_{n-1}-x_{n}\right)\right\| \\
& \leq\left\|\nabla h\left(x_{n-1}-x_{n}\right)\right\| \rightarrow 0 \quad \text { when } \mathrm{n} \rightarrow \infty . \\
\Rightarrow & \lim _{n \rightarrow \infty}\left\|\nabla h\left(x_{n}\right)-\nabla h\left(z_{n}\right)\right\|=0 .
\end{aligned}
$$

Invoking [4], we obtain

$$
\lim _{n \rightarrow \infty}\left\|x_{n}-z_{n}\right\|=0 \text {. }
$$

This establishes (ii).

Moreso, since $\left\{z_{n}\right\}$ is bounded and using P6, (21) we obtain

$\lim _{n \rightarrow \infty} d_{h}\left(x_{n}, z_{n}\right)=0$.

In addition, since $x_{n+1} \in K_{n+1} \subset K_{n}$, from the definition of the half space, we obtain

$$
d_{h}\left(x_{n+1}, w_{n}\right) \leq d_{h}\left(x_{n+1}, z_{n}\right)
$$

Moreover, using P4, (17), (20), and (2), we get

$$
\lim _{n \rightarrow \infty} d_{h}\left(x_{n+1}, z_{n}\right)=0
$$

This implies that

$$
\lim _{n \rightarrow \infty} d_{h}\left(x_{n+1}, w_{n}\right)=0 .
$$

Thus, [7] suggest that 
and $\quad \lim _{n \rightarrow \infty}\left\|x_{n+1}-z_{n}\right\|=0$

$\lim _{n \rightarrow \infty}\left\|x_{n+1}-w_{n}\right\|=0$.

This establishes (iii).

Combining (18) and (26), we get

$\left\|x_{n}-w_{n}\right\| \rightarrow 0$ as $\mathrm{n} \rightarrow \infty$.

Also, from (26) we get

$\lim _{n \rightarrow \infty}\left\|x_{n}-w_{n}\right\|=0$

and

$\lim _{n \rightarrow \infty}\left\|z_{n}-w_{n}\right\|=0$.

This establishes (iv).

Next, using [13], this implies

$$
\begin{aligned}
& d_{h}\left(y_{n}, w_{n}\right)=d_{h}\left(y_{n}, T_{r_{n}}^{a} y_{n}\right) \leq d_{h}\left(u, w_{n}\right)-d_{h}\left(u, y_{n}\right) \\
& \leq d_{h}\left(u, y_{n}\right)-d_{h}\left(u, y_{n}\right) \rightarrow 0 \text { as } \mathrm{n} \rightarrow \infty . \\
\Rightarrow & \lim _{n \rightarrow \infty} d_{h}\left(y_{n}, w_{n}\right)=0 .
\end{aligned}
$$

Using [7] gives

$$
\lim _{n \rightarrow \infty}\left\|w_{n}-y_{n}\right\|=0 \text {. }
$$

This establishes (v).

Now, from the uniform continuity of $\nabla h$, (29) becomes

$$
\left\|\nabla h\left(w_{n}\right)-\nabla h\left(y_{n}\right)\right\| \rightarrow 0 \text { as } n \rightarrow \infty .
$$

Thus using (29) with (27) gives

$$
\lim _{n \rightarrow \infty}\left\|x_{n}-y_{n}\right\|=0 \text {. }
$$

This establishes (vi).

Using (26) with (29) gives

$$
\lim _{n \rightarrow \infty}\left\|x_{n+1}-y_{n}\right\|=0 \text {. }
$$

With this and P6 we get

$$
d_{h}\left(x_{n+1}, y_{n}\right) \rightarrow 0 \text { as } n \rightarrow \infty \text {. }
$$

Thus combining (29) and (28) we get

$$
\lim _{n \rightarrow \infty}\left\|z_{n}-y_{n}\right\|=0
$$


Since $\nabla h$ is uniformly continuous, we obtain

$$
\left\|\nabla h\left(z_{n}\right)-\nabla h\left(y_{n}\right)\right\| \rightarrow 0 \text { as } n \rightarrow \infty
$$

Since, $y_{n}=\nabla h^{*}\left((1-\eta) \nabla h\left(z_{n}\right)+\eta \nabla h\left(G z_{n}\right)\right)$, we obtain

$$
\left\|\nabla h\left(z_{n}\right)-\nabla h\left(y_{n}\right)\right\|=\left\|\nabla h\left(z_{n}\right)-\nabla h\left(z_{n}\right)+\eta\left(\nabla h\left(G z_{n}\right)-\nabla h\left(z_{n}\right)\right)\right\|=\eta\left\|\nabla h\left(G z_{n}\right)-\nabla h\left(z_{n}\right)\right\| .
$$

Using (33) gives

$$
\left\|\nabla h\left(G z_{n}\right)-\nabla h\left(z_{n}\right)\right\| \rightarrow \text { as } n \rightarrow \infty \text {. }
$$

So that

$$
\left\|z_{n}-G z_{n}\right\| \rightarrow 0 \text { as } n \rightarrow \infty
$$

This establishes (vii).

Theorem 2.3. Let $K$ be a non-void, closed and convex subset of reflexive Banach space $X$. Let the function $h: X \rightarrow(-\infty,+\infty)$ represent bounded Legendre, uniform Fréchet differentiable, totally convex. Let the map $G: K \rightarrow K$ represent a Bregman relatively nonexpansive, $a: K \rightarrow X^{*}$ represent a continuous monotone map. We assume $F=\operatorname{Fix}(G) \bigcap \operatorname{VI}(K, a)$ to be non-void. Let $\left\{x_{n}\right\}$ be produced by (11). Then $\left\{x_{n}\right\}$ converge strong to a point of $F=F i x(G) \bigcap V I(K, a)$.

\section{Proof.}

From Lemma 2.2, $\left\{x_{n}\right\}$ is bounded. Since $X$ is reflexive, there exist $\left\{x_{n_{i}}\right\}$ of $\left\{x_{n}\right\}$ such that $x_{n_{i}}$ converges weakly to $\hat{u}$ as $i \rightarrow \infty$. We now show that $\hat{u}=P_{F}^{h}\left(x_{0}\right)$. Setting $\hat{q}=P_{F}^{h}\left(x_{0}\right)$, then from (4), we get

$$
d_{h}\left(\hat{u}, x_{0}\right) \leq d_{h}\left(u, x_{0}\right) \quad \forall u \in F \text { and } d_{h}\left(\hat{q}, x_{0}\right) \leq d_{h}\left(u, x_{0}\right) \quad \forall u \in F \text {, }
$$

in addition to (35), we get

$$
\begin{aligned}
d_{h}(\hat{u}, \hat{q}) & \leq d_{h}\left(\hat{u}, x_{0}\right)-d_{h}\left(\hat{q}, x_{0}\right) \\
& \leq d_{h}\left(u, x_{0}\right)-d_{h}\left(u, x_{0}\right) \\
& =0 .
\end{aligned}
$$

This implies

$$
d_{h}(\hat{u}, \hat{p}) \leq 0 .
$$

By the uniqueness of $P_{F}^{h}\left(x_{0}\right), \hat{u}=\hat{p}$. So, we have shown that $\hat{u}=P_{F}^{h}\left(x_{0}\right)$. Next, we show that $x_{n} \rightarrow \hat{u}=P_{F}^{h}\left(x_{0}\right)$ as $n \rightarrow \infty$. To do this, recall from Lemma 2.1, that the half space $K_{n}$ is closed and convex and $F \subset K_{n}$, implying that $K_{n}$ is weakly closed and $\hat{u} \in K_{n}, \forall n \geq 0$. Recall from definition that $x_{n_{i}}=P_{K_{n_{i}}}^{f}\left(x_{0}\right)$, so that $d_{h}\left(x_{n_{i}}, x_{0}\right) \leq d_{h}\left(\hat{u}, x_{0}\right)$. Using the weakly lower semi-continuity of $h$ on the convex set, we get 


$$
\begin{aligned}
& d_{h}\left(\hat{u}, x_{0}\right)=h(\hat{u})-h\left(x_{0}\right)-\left\langle\nabla h\left(x_{0}\right), \hat{u}-x_{0}\right\rangle \\
& \leq \liminf _{i \rightarrow \infty}\left\{h\left(x_{n_{i}}\right)-h\left(x_{0}\right)-\left\langle\nabla h\left(x_{0}\right), x_{n}-x_{0}\right\rangle\right\} \\
& =\liminf _{i \rightarrow \infty} d_{h}\left(x_{n_{i}}, x_{0}\right) \\
& \leq \limsup _{i \rightarrow \infty} d_{h}\left(x_{n_{i}}, x_{0}\right) \\
& \leq d_{h}\left(\hat{u}, x_{0}\right) .
\end{aligned}
$$

This implies

$$
\lim _{i \rightarrow \infty} d_{h}\left(x_{n_{i}}, x_{0}\right)=d_{h}\left(\hat{u}, x_{0}\right)
$$

This implies that $\lim _{i \rightarrow \infty} h\left(x_{n_{i}}\right)=h(\hat{u})$. Since $h$ is uniformly continuous, we get

$$
\lim _{i \rightarrow \infty} x_{n_{i}}=\hat{u} .
$$

Since $\left\{x_{n}\right\}$ is convergent, invoking Lemma 2.2 gives

$$
x_{n} \rightarrow \hat{u} \text { as } n \rightarrow \infty
$$

Now from Lemma 2.2, $\left\{z_{n}\right\}$ is bounded implying there exists a subsequence $\left\{z_{n_{i}}\right\}$ such that $z_{n_{i}}$ converges weakly to $\hat{u}$ as $i \rightarrow \infty$. Applying condition (ix) in Lemma 2.2, we obtain $\lim _{i \rightarrow \infty}\left\|z_{n_{i}}-G z_{n_{i}}\right\|=0$. Since our map is Bregman relatively nonexpansive, we have $\hat{u} \in F(G)$. Next, we show that $\hat{u} \in V I(K, a)$. From the definition of $w_{n}$, we get

$$
\left\langle a w_{n}, z\right\rangle r_{n}-\left\langle a w_{n}, w_{n}\right\rangle r_{n}+\left\langle\nabla h\left(w_{n}\right)-\nabla h\left(z_{n}\right), z-w_{n}\right\rangle \geq 0, \forall z \in K,
$$

using (39), the fact that $w_{n}$ converges to $\hat{u}$ as $n \rightarrow \infty$, and the continuity of $a$, we have

$$
\langle a \hat{u}, z\rangle-\langle a \hat{u}, \hat{u}\rangle \geq 0 \quad \forall z \in K \text {. }
$$

Thus $\hat{u} \in V I(K, a)$. Therefore, $\hat{u} \in F=\operatorname{Fix}(G) \bigcap V I(K, a)$.

Remark 2.4. Our result in particular, extends the mappings and results of [14] to a more general mapping corresponding to Bregman distance function in reflexive Banach space. Our scheme has the inertial term known to speed up convergence of sequences. Our scheme is applicable in Hilbert spaces when we consider $\nabla h=I$, the identity mapping and $X=H$.

\section{Numerical Example}

A direct application of Theorem 2.3 is in this section given to demonstrate convergence of sequences generated by it.

Let $X=R, K=[0,4], h(x)=x^{2}, \nabla h(x)=2 x, h^{*}\left(u^{*}\right)=\sup \left\{\left\langle u^{*}, x\right\rangle-h(x)\right\}=\frac{1}{4}\left(u^{*}\right)^{2}$,

$\nabla h^{*}\left(u^{*}\right)=\frac{1}{2} u^{*} \cdot\langle a z, y-z\rangle=z y-z^{2}$.

Let $G:[0,4] \rightarrow[0, \infty)$ be defined by 


$$
G(x)=\left\{\begin{array}{l}
0 \text { if } x \neq 4 \\
2 \text { if } x=4
\end{array}\right.
$$

It is clear that $\operatorname{Fix}(G)=\{0\}$ since for $x \neq 4, G(x)=x \Rightarrow 0=x$. Thus, for any $x \neq 4, x=0$. Again, for $x=4, G(x)=x \Rightarrow 2=x$. Thus for $x=4,2=4$, which is not possible. So Fix $(G)=\{0\}$. Next, we observe that $x_{n}$ converges weakly to 0 , hence $x_{n}-G x_{n} \rightarrow 0$ and $\operatorname{Fi} \hat{x}(G)=F i x(G)=\{0\}$ [15]. From the definition of Bregman relatively nonexpansive mapping, one can easily demonstrate that

$$
d_{h}(0, G x) \leq d_{h}(0, x)
$$

Infact

$$
\begin{aligned}
d_{h}(0, G x) & =h(0)-h(G x)-\langle\nabla h(G x), 0-G x\rangle \\
& =0-4-\langle 0,0-2\rangle \\
& =-4 \\
d_{h}(0, x)= & h(0)-h(x)-\langle\nabla h(x), 0-x\rangle \\
= & 0-x^{2}-\langle 2 x, 0-x\rangle \\
= & -x^{2}+2 x^{2} \\
= & x^{2} .
\end{aligned}
$$

Thus, using (40) and (41) we have

$$
d_{h}(0, G x) \leq d_{h}(0, x), \quad \text { forall } x \in[0,4] \text {. }
$$

Furthermore, setting $x_{0}=\frac{1}{2}, x_{1}=1, \alpha_{n}=\frac{n+1}{4 n}, \eta=\frac{1}{2}, r=1$, we get

$$
\begin{aligned}
& z_{n}=\nabla h^{*}\left(\nabla h\left(x_{n}\right)+\frac{n+1}{4 n}\left(\nabla h\left(x_{n}\right)-\nabla h\left(x_{n-1}\right)\right)\right) \\
& y_{n}=\nabla h^{*}\left((1-\eta) \nabla h\left(z_{n}\right)+\eta \nabla h\left(G z_{n}\right)\right)=\frac{1}{2} z_{n}, \\
& w_{n}=T_{r_{n}}^{a} y_{n}=\frac{1}{3} z_{n} . \\
& K_{n+1}=\left\{u \in K_{n}: d_{h}\left(u, w_{n}\right) \leq d_{h}\left(u, z_{n}\right)\right\}=\left\{u \in K_{n}: u \leq-\frac{13}{24} z_{n}\right\} .
\end{aligned}
$$

Therefore, we have our scheme (11) now simplified thus: 


$$
\left\{\begin{array}{l}
x_{0} \in[0,4], \text { Chosen arbitrarib, } \\
z_{n}=\nabla h^{*}\left(\nabla h\left(x_{n}\right)+\frac{n+1}{4 n}\left(\nabla h\left(x_{n}\right)-\nabla h\left(x_{n-1}\right)\right)\right), \\
y_{n}=\frac{1}{2} z_{n}, \\
w_{n}=\frac{1}{3} z_{n}, \\
K_{n+1}=\left\{u \in K_{n}: u \leq-\frac{13}{24} z_{n}\right\} \\
x_{n+1}=P_{K_{n+1}}^{h}\left(x_{0}\right)=u, \quad \forall n \geq 1 .
\end{array}\right.
$$

\section{CONFLICTS OF INTEREST}

No conflict of interest was declared by the authors.

\section{REFERENCES}

[1] Bertsekas, D.P., “Convex optimization theory”, Anthena Scientific, (2009).

[2] Reich, S., Sabach, S., "Existence and approximation of fixed points of Bregman firmly nonexpansive mappings in reflexive Banach spaces", Fixed-point Algorithms for Inverse Problems in Science and Engineering, 49: 301-316, (2011).

[3] Reich, S., Sabach, S., "A strong convergence theorem for a proximal-type algorithm in reflexive Banach spaces", Journal of Nonlinear Convex Analysis, 10(3): 471-485, (2009).

[4] Butnariu, D., Resmerita, E., "Bregman distances, totally convex functions and a method for solving operator equations in Banach spaces", Abstract and Applied Analysis, 2006:1-39, (2006).

[5] Naraghirad, E., Yao, J. C., "Bregman weak relatively nonexpansive mappings in Banach spaces", Fixed Point Theory and Applications, 2013(141): 1-43, (2013).

[6] Alber, Y. I., "Metric and generalized projection operators in Banach Spaces: Properties and Applications", Lecture Notes in Pure and Applied Mathematics, 15-50, (1996).

[7] Butnariu, D., Iusem, A. N., "Totally Convex Functions for Fixed Points Computation and Infinite Dimensional Optimization”, Kluwer Academic, Dordrecht, 40: (2000).

[8] Bauschke, H. H., Borwein, J. M., Combettes, P. L., "Essential smoothness, essential strict convexity, and Legendre functions in Banach spaces", Communications in Contemporary Mathematics, 3(4): 615-647, (2001).

[9] Bonnas, J. F., Shapiro, A., "Perturbation Analysis of Optimization Problems", Springer, New York, (2000).

[10] Phelps, R. P., “Convex Functions, Monotone Operators and Differentiability”, Springer, Berlin, 1364: (1993).

[11] Rockafellar, R. T., "Convex Analysis”, Princeto University Press, Princeton, (1970).

[12] Zalinescu, C., "Convex Analysis in General Vector Spaces", World Scientific, River Edge, NJ, USA, (2002). 
[13] Alghamdi, M. A., Shahzad, N., Zegeye, H., "Fixed points of Bregman relatively nonexpansive mappings and solutions of variational inequality problems", Journal of Nonlinear Science and Application, 9: 2541-2552, (2016).

[14] Inoue, G., Takahashi, W., Zembayashi, K., "Strong convergence theorems by hybrid method for maximal monotone operators and relatively nonexpansive mappings in Banach spaces", J. Convex Anal., 16: 791-806, (2009).

[15] Naraghirad, E., "Halpern's iteration for Bregman relatively nonexpansive mappings in Banach spaces", Numerical Functional Analysis and optimization, 34(10): 1129-1155, (2013).

[16] Chidume, C.E., Ikechukwu, S.I., Adamu, A., "Inertial algorithm for approximating a common fixed point for a countable family of relatively nonexpansive maps", Fixed Point Theory and Applications, 2018(9): 1-9, (2018).

[17] Dong, Q. L., Cho, Y, J., Rassias, Th. M., "General Inertial Mann Algorithms and their convergence analysis for nonexpansive mappings", Applications of Nonlinear Analysis, Springer Optimization and its Applications, 134: (2018).

[18] Polyak, B.T., "Some methods of speeding up the convergence of iteration methods", USSR Comput. Math. and Math. Phys., 4(5): 1-17, (1964). 